\title{
The gauge dual of Romans mass
}

\author{
Davide Gaiotto ${ }^{1}$ and Alessandro Tomasiello ${ }^{2,3}$ \\ ${ }^{1}$ School of Natural Sciences, Institute for Advanced Study, Princeton, NJ 08540, USA \\ 2 Jefferson Physical Laboratory, Harvard University, Cambridge, MA 02138, USA \\ ${ }^{3}$ Università di Milano-Bicocca and INFN, sezione di Milano-Bicocca, I-20126 Milano, Italy
}

\begin{abstract}
We deform the recently proposed holographic duality between the ABJM $\mathcal{N}=6$ ChernSimons-matter theory and type IIA string theory in $\mathrm{AdS}_{4} \times \mathbb{C P}^{3}$. We add a non-zero Romans mass $F_{0}$, whose dual we identify as the sum of the Chern-Simons levels for the two gauge groups. One can naturally identify four different theories, with different amounts of supersymmetry and of flavor symmetry.
\end{abstract}




\section{Introduction}

The gauge/gravity duality proposed recently in [1] (ABJM) is the first example in which the conformal field theory (CFT) is a three-dimensional theory with an explicit Lagrangian description. The $\mathrm{CFT}_{3}$ is a Chern-Simons theory coupled to matter fields. There are two gauge groups, with Chern-Simons levels $k$ and $-k$. The gravity duals are old solutions [2, 3] that involve internal fluxes $F_{2}$ and $F_{6}$.

Using the Chern-Simons action to write down a Lagrangian for a CFT is a very natural idea. Indeed, in three dimensions, the usual Yang-Mills action would involve a dimensionful coupling constant. In contrast, the Chern-Simons action involves a dimensionless parameters, which is even an integer for quantum-mechanical reasons. Although the Chern-Simons theory is by itself topological, and it hence describes only a finite number of degrees of freedom, it can be coupled to scalars and fermions to achieve scale invariance.

It was initially expected that such Chern-Simons-matter actions would be dual to $\mathrm{AdS}_{4}$ solutions in string theory involving Romans mass $F_{0}$ [4]. This is because of the coupling $\int_{\mathrm{D} 2} F_{0} C S(a)$, where $a$ is the world-volume gauge field on the D2.

The duals found in [1] confounded this expectation, in that no $F_{0}$ is present in the solution, even though the theory contains a Chern-Simons action. The reason for this is, roughly, that the internal flux $F_{2}$ also induces a Chern-Simons coupling on "fractional D2's" - D4's wrapping a vanishing two-cycle.

This does not, however, invalidate the original reason to expect that $F_{0}$ would induce a Chern-Simons action. In fact, supersymmetric solutions with non-zero Romans mass were found recently in [5], and with an internal space whose topology is $\mathbb{C P}^{3}$, the same as in the $\mathcal{N}=6$ solutions [2, 3]. Since these solutions have only $\mathcal{N}=1$ supersymmetry, one might be skeptical of any proposed gauge duals to them. However, the solutions in [5] happen to have a parameter space that, although discretized by flux quantization, gets arbitrarily close to the $\mathcal{N}=6$ solutions of [2, 3].

Exploiting this fact, in section 3.2 we will be able to find Chern-Simons-matter theories which are, in a sense, small deformations of the ABJM theory, and that should be dual to the solutions in [5]. These duals vindicate the expectation mentioned above, that the Romans mass should contribute to the Chern-Simons levels. In fact, both $F_{0}$ and $F_{2}$ contribute to the Chern-Simons actions for the two gauge groups: morally, one gauge group has level $k$, and the other has level $F_{0}-k$, where $k=\int_{\mathbb{C P}^{1}} F_{2}$. See also (2.1) and (4.19) below.

In fact, in section 2 we will show that this is a general feature, dictated by considera- 
tions involving brane probes and simple but robust topological arguments.

Also, we will see in section 3 that similar ideas to the ones that lead to the definition of the $\mathcal{N}=1$ field theory in 3.2 also suggest how to define theories with $\mathcal{N}=0,2$ and 3. At the same time, the $\mathrm{SO}(6) \mathrm{R}$-symmetry of the $\mathcal{N}=6$ theory gets broken in various ways, summarized in table 3 .

This means that the situation is now reversed: it is now field theory that suggests where to look for gravity solutions. We discuss these duals in section 4 . The $\mathcal{N}=1$ solutions, as we mentioned, were found in [5], and we review them in section 4.2, but the solutions with $\mathcal{N}=0,2$ and 3 seem to be new. Perhaps surprisingly, the ones which are easiest to find are the solutions with $\mathcal{N}=0$, as we will see in section 4.1. This is because they have the highest amount of isometries (as one can see from table 33). We have not found yet 1 the solutions with $\mathcal{N}=2$ and 3 , but we know a few features that they should have, as we discuss in sections 3.3 and 3.4. Work is in progress to find them explicitly.

\section{Romans mass as sum of Chern-Simons levels}

The Chern-Simons-matter theory found in [1], to be reviewed more thoroughly in section 3, has two gauge groups, with CS levels $k_{1}=-k_{2} \equiv-k$. It is the dual to a certain IIA solution on $\mathrm{AdS}_{4} \times \mathbb{C P}^{3}$; we will refer to it as the "N$=6$ solution" from now on. Suppose one has a solution obtained by a small perturbation of this $\mathcal{N}=6$ solution and involving a non-zero Romans mass $F_{0}$. In this section, we will argue rather generally that the dual theory to such a solution is a perturbation of the ABJM theory with levels

$$
k_{1}+k_{2}=F_{0}
$$

The argument relies on the field-operator correspondence in AdS/CFT. In particular, we want to consider the field theory duals to particles in AdS obtained by wrapping Dbranes on subspaces of the internal $\mathbb{C P}^{3}$. Although the argument ultimately only needs D0 branes, we will start by reviewing some background material.

First of all, let us recall the better-known case of $\mathrm{AdS}_{5} \times S^{5}$ [8]. In that case, the $N$ quanta of $F_{5}$ on the sphere induces a tadpole $\int a F_{5}=N \int a$ for the $\mathrm{U}(1)$ gauge field

\footnotetext{
${ }^{1}$ There exist $\mathcal{N}=2$ solutions with an internal space whose topology is $\mathbb{C P}^{3}$, but they are vacua of the same effective theory that also describes the $\mathcal{N}=6$ solutions; hence, they cannot involve $F_{0} \neq 0$. Threedimensional analogues of the four-dimensional "beta-deformed" theories [6] might also be considered, as done for example in [7, but such theories should be continuous deformations of the $\mathcal{N}=6$ theory, and hence should have the same Chern-Simons levels.
} 
on a D5 brane wrapping the $S^{5}$. This tadpole requires $N$ fundamental strings to end on the $D 5$ brane, which is then identified with a "baryonic operator", i. e. $N$ fundamental Wilson lines ending on an $\epsilon$ tensor.

In the $\mathrm{ABJM}$ case, there is a richer story. The $\mathcal{N}=6$ solution on $\mathrm{AdS}_{4} \times \mathbb{C P}^{3}[2,3]$ is characterized by two flux integers, not one: the integrals of $F_{2}$ and $F_{6}, k \equiv \int_{\mathbb{C P}^{1}} F_{2}$ and $N \equiv \int_{\mathbb{C P}^{3}} F_{6}$. In our notation (to be reviewed more extensively in section 4.2), these fluxes are related to the overall radius $R$ in string units and to the string coupling $g_{s}$ by

$$
k=\frac{\pi R}{g_{s}}, \quad N=\frac{\pi^{3} R^{5}}{2 g_{s}} .
$$

In this solution, there are now two types of branes with tadpoles. A D6 brane wrapping the whole $\mathbb{C P}^{3}$ would have a tadpole of $N$ units, require $N$ strings to end on it, and correspond to $N$ Wilson lines ending on an $\epsilon$ tensor. This baryonic vertex is quite similar to the one in $\mathrm{AdS}_{5} \times S^{5}$.

Another brane with a tadpole is a D2 brane wrapping a $\mathbb{C P}^{1}$; such a brane has $k$ units of $\mathrm{U}(1)$ charge on its worldvolume. In this case, the $k$ Wilson lines cannot end on an $\epsilon$ tensor. However, in a Chern-Simons theory at level $k$, a Wilson line in the representation $\operatorname{Sym}_{k}$ (the one obtained by symmetrizing $k$ fundamentals), extended say from infinity to a point $p$, is equivalent [9] to a monopole ('t Hooft) operator creating one unit of flux around the point $p$. In other words, only the endpoint of the Wilson line is physical. Hence, we can have $k$ Wilson line end in a point, and this is the field theory dual to $k$ fundamental strings ending on a D2 brane.

On the other hand, there are also branes that do not have any tadpoles on their worldvolumes. For example, this is the case for D4 branes. They have been mapped to the so-called dibaryons, made of $N$ bifundamental fields $X_{A}$ attached to two $\epsilon$ tensors. Notice that the mass of a D4 goes indeed like $m_{D 4} R \sim R^{5} / g_{s} \sim N$.

Finally, D0 branes also have no tadpole, and correspond to di-monopole operators, with charge $(1,1)$ under the two gauge groups. Once again following [9], because of the Chern-Simons couplings of the two gauge groups, these operators are equivalent to Wilson lines in the representation $\left(\mathrm{Sym}_{k}, \overline{\mathrm{Sym}_{k}}\right)$. In other words, these operators carry both $k$ fundamental indices of one group, and $k$ anti-fundamentals of the other group. These indices can be saturated by $k$ bifundamental $X_{A}$ fields. The mass of a D0 goes like $m_{D 0} R \sim R / g_{s} \sim k$, which again makes sense.

This completes our list of duals to D-branes for the original ABJM duality. Let us now see how we can modify it. A simple modification of the background consists in adding closed $B$-field. In general, the most sensible definition of flux is the so-called 
"Page charge", the integral on a $p$-cycle of $\tilde{F}_{p} \equiv\left(e^{B} F\right)_{p}$. For example, one can generate in this situation a $\tilde{F}_{4}=B F_{2}$ that can have a non-zero flux $n_{4}$. In such a background, the D4 brane has now $n$ units of $\mathrm{U}(1)$ tadpole (because $\tilde{F}_{4}$ is the flux coupling to the worldvolume gauge field). We saw before that the dual to the $\mathrm{D} 4$ when $n_{4}=0$ was the dibaryon operator. Now, for $n_{4} \neq 0$, the tadpole is telling us that this di-baryon operator has now $n$ "dangling" fundamental indices on one of the two sides that have been contracted with $\epsilon$ tensors. This suggests [10] that the theory has now changed: the two ranks should now differ by $n_{4}$.

One can now try to apply the same reasoning to the D0. We just saw an easy way to modify the ABJM background by adding $\tilde{F}_{4}$ flux. 2 Let us now suppose we have a way of deforming the background and of introducing a non-zero Romans mass $F_{0}$; we have not shown how to do this at this point, but we will see it later. Now a D0 brane will develop a tadpole, because of the coupling $\int a F_{0}$, where $a$ is the worldvolume $\mathrm{U}(1)$ field. This means that one needs $F_{0}$ fundamental strings ending on the D0. This can be explained with the two Chern-Simons levels being no longer equal. Indeed, in such a theory a di-monopole operator of charge $(1,1)$ has $k_{1}$ indices in the fundamental of the first gauge group, and $-k_{2}$ indices in the anti-fundamental of the second gauge group. Even if one dresses this operator up with $k_{2}$ bifundamental fields, one is left with $k_{1}+k_{2}$ "dangling" fundamental indices. These indices are in correspondence with the $F_{0}$ fundamental strings above. This leads us to conclude (2.1).

We can strengthen this conclusion by analyzing the field theory dual of configurations where a single D2 brane, point-like in $\mathbb{C P}^{3}$, acts as a domain wall in $A d S_{4}$. These configurations, which we will also consider in sections 3.3 and 3.4, correspond in the ABJM theory to vacua where the bifundamental fields $X^{I}$ in a single $U(1) \times U(1)$ block are given large expectation values. The relevant terms in the Lagrangian are

$$
k_{1} C S\left(a_{1}\right)+k_{2} C S\left(a_{2}\right)+|X|^{2}\left(a_{1}-a_{2}\right)^{2}
$$

The bifundamental fields are charged under the difference of the two $U(1)$ gauge fields $a_{-}=a_{1}-a_{2}$, which becomes very massive. At low energy, the two gauge fields are forced to be equal.

If the Chern-Simons levels are equal and opposite, the Chern-Simons actions cancel each other. The dynamics of the surviving gauge field $a_{+}=a_{1}+a_{2}$ is controlled by a YangMills term generated by integrating away the difference between the gauge fields [11].

\footnotetext{
${ }^{2}$ Incidentally, one cannot add an untilded $F_{4}$ without also adding $F_{0}$.
} 
Indeed the Lagrangian can be rewritten schematically as

$$
k a_{-} \wedge d a_{+}+|X|^{2} a_{-}^{2}
$$

and $a_{-}$integrated out explicitly to give

$$
\frac{k^{2}}{|X|^{2}}\left(d a_{+}\right)^{2}
$$

$a_{+}$matches well the gauge field living on the D2 brane domain wall.

If the Chern-Simons levels are changed to more generic values, the light gauge field $a=a_{1}=a_{2}$ will have a residual $k_{1}+k_{2}$ Chern-Simons coupling. This matches the

above mentioned coupling $\int_{D 2} F_{0} C S(a)$ on a D2 brane in the presence of Roman mass if $F_{0}=k_{1}+k_{2}$.

Having reached this general conclusion, we now have to ask ourselves whether one can define CFTs by deforming the ABJM theory by letting the ranks not sum to zero, and - dually - whether there are any supergravity solutions that deform the $\mathcal{N}=6$ solution by a small non-zero Romans mass $F_{0}$. We will consider these two questions in sections 3 and 4 .

\section{A hierarchy of theories with unequal levels}

In this section, we start from the ABJM theory and try to let the levels of the two gauge groups not sum to zero any more: $k_{1} \neq-k_{2}$.

This is a bit ambiguous, however. By supersymmetry, the level appears in several terms in the ABJM action, such as the bosonic potential and the fermion-boson couplings. How many of those coefficients we change is up to us. As we will explain in this section, we can choose to preserve different amounts of supersymmetry and different global symmetries. The theories we will define are summarized in table 3 .

We will argue that there is a CFT in each of these classes of theories. In other words, for $k_{1} \neq-k_{2}$, the ABJM fixed point of the RG flow splits in four different fixed points, each with a different amount of supersymmetry and global symmetries.

As we will see, there is more control on the field theory side as supersymmetry increases; on the gravity side, to be discussed in section 4, there is more control as the amount of bosonic symmetry increases. 


\begin{tabular}{|c||c|c|}
\cline { 2 - 3 } \multicolumn{1}{l||}{} & supersymmetry & global symmetry \\
\hline \hline Sec. 3.1 & $\mathcal{N}=0$ & $\mathrm{SO}(6)$ \\
\hline Sec. 3.2 & $\mathcal{N}=1$ & $\mathrm{SO}(5)$ \\
\hline Sec. 3.3 & $\mathcal{N}=2$ & $\mathrm{SO}(2)_{\mathrm{R}} \times \mathrm{SO}(4)$ \\
\hline Sec. 3.4 & $\mathcal{N}=3$ & $\mathrm{SO}(3)_{\mathrm{R}} \times \mathrm{SO}(3)$ \\
\hline
\end{tabular}

Table 1: The various theories with $k_{1} \neq-k_{2}$ that we will define in this section. The subscript $\mathrm{R}$ denotes $\mathrm{R}$-symmetry.

\section{$3.1 \mathcal{N}=0$}

One can start by simply taking the ABJM written down in components (as can be found for example in [12, Sec. 4]), and changing the Chern-Simons levels, so that now $k_{1} \neq-k_{2}$ - and nothing else.

Once we do this, the theory is not conformal any more. However, the RG flow will respect the $\mathrm{SO}(6)$ symmetry of the action. In other words, the flow will happen in the finite-dimensional space of theories $\operatorname{QFT}_{\mathcal{N}=0}\left(k_{1}, k_{2}\right)$, defined as the space of ChernSimons-matter theories with the same field content as the ABJM, with Chern-Simons levels $k_{1}$ and $k_{2}$ for the two gauge groups, and with $\mathrm{SO}(6)$ symmetry. For example, the most general single-trace potential will have the form

$$
V=c_{I J K}^{L M N} \operatorname{Tr}\left[X^{I} X_{L}^{\dagger} X^{J} X_{M}^{\dagger} X^{K} X_{N}^{\dagger}\right],
$$

where we have collected together all the fields in $X_{I}=\left(A_{1}, A_{2}, B_{1}^{\dagger}, B_{2}^{\dagger}\right)$. The coefficients $c_{I J K}^{L M N}$ are some of the coordinates on the space of theories $\mathrm{QFT}_{\mathcal{N}=0}\left(k_{1}, k_{2}\right)$. More generally, let us denote by $c_{O}$ the coefficient multiplying the operator $O$; besides the terms in the bosonic potential (3.1), there are quartic fermion-boson couplings, and possible doubletrace terms and triple-trace terms that we will discuss in due course. We will denote by $c_{O}^{0}$ the value of the coefficient $c_{O}$ in the ABJM theory.

We now want to argue that there will still be a CFT in this space of theories, if $k_{1}+k_{2}$ is small in a sense that we will specify.

Quantities as the $\beta$ functions of the $c_{O}$ will be functions of the two t'Hooft couplings $\lambda_{i}=\frac{N}{k_{i}}$. For Chern Simons theories, these t'Hooft couplings are not subject to RG flow. We are interested in a regime where $\lambda_{+}=\lambda_{1}+\lambda_{2}$ is kept small, while $\lambda_{-}=\lambda_{1}-\lambda_{2}$ may 
be large.

If we change the levels and no other coefficient in the action, so that $c_{O}=c_{O}^{0}$, we expect that the beta functions will not change much with respect to the ABJM case, where they are 0.

$$
\beta_{O}=\left(\lambda_{1}+\lambda_{2}\right) \delta \beta_{O}
$$

Essentially, we are assuming that the $\beta$ functions should be analytic in $\lambda_{+}$around $\lambda_{+}=0$, even for large $\lambda_{-}$. In general $\delta \beta$ will not be zero, and the theory will not be conformal. However, we can respond by changing also the coefficient $c_{O}$ a bit. The variation of the beta function is related to the anomalous dimension of the operator (for example, a marginal operator can be added infinitesimally to a CFT without changing the beta functions at the first order). Hence we can write:

$$
\beta_{O}=\left(\lambda_{1}+\lambda_{2}\right) \delta \beta_{O}+\delta c_{O} \gamma_{O}
$$

where $\delta c_{O}=c_{O}-c_{O}^{0}$ Now, if $\gamma_{O} \neq 0$, we can just take

$$
\delta c_{O}=-\left(\lambda_{1}+\lambda_{2}\right) \frac{\delta \beta_{O}}{\gamma_{O}}
$$

and obtain $\beta_{O}=0$ again.

Hence, in the space $\mathrm{QFT}_{\mathcal{N}=0}\left(k_{1}, k_{2}\right)$, there will be a conformal theory that we will denote $\mathrm{CFT}_{\mathcal{N}=0}\left(k_{1}, k_{2}\right)$, with Chern-Simons action with levels $k_{1} \neq-k_{2}$, and the rest of the action of the form $\sum_{O}\left(c_{O}+\delta c_{O}\right) O$.

There are a few things to remark about this logic. First of all, looking at (3.4), we can now say in what sense $\lambda_{1}+\lambda_{2}$ needs to be small: we have to assume

$$
\left(\lambda_{1}+\lambda_{2}\right) \delta \beta_{O} \ll \gamma_{O}
$$

for all $O$ that have $\mathrm{SO}(6)$ symmetry. At weak coupling, this means that $k_{1}+k_{2} \ll k_{1}, k_{2}$. At strong coupling, we expect that $\left(\lambda_{1}+\lambda_{2}\right)=\frac{k_{1}+k_{2}}{k_{1}-k_{2}} \lambda_{-} \ll 1$ to be sufficient, though it is possible that the detailed $\lambda_{-}$dependence of $\delta \beta_{O}, \gamma_{O}$ will require a more stringent $\frac{k_{1}+k_{2}}{k_{1}-k_{2}} \lambda_{-}^{C} \ll 1$ for some constant $C>1$.

Second, our reasoning works only if

$$
\gamma_{O} \neq 0
$$

again for all $O$ that are $\mathrm{SO}(6)$ singlets. If the operator $O$ is marginal in the ABJM theory, $\gamma_{O}$ will also be proportional to $\lambda_{+}$, and any attempt to set the beta function to zero will require a large $\delta c_{O}$, outside the range of our perturbative approximation. 
It is easy to write down an operator of classical dimension 3, but most of these will acquire an anomalous dimension in the ABJM theory. The ones that remain marginal are the ones that are protected by supersymmetry. To find these, one can determine the chiral primaries for the ABJM theory. The single trace ones are of the form $\operatorname{Tr}\left(X^{\left(I_{1}\right.} X_{\left(J_{1}\right.}^{\dagger} X^{I_{2}} X_{J_{2}}^{\dagger} \ldots X^{\left.I_{n}\right)} X_{J_{n}}^{\dagger}\right)$, where the indices up and down are separately symmetrized. This is the representation $(n, 0, n)$ of $\mathrm{SU}(4)$. (This result is similar to the computation of chiral primaries for the super Yang-Mills theory in four dimensions, that are in the $(0, n, 0)$ of $\mathrm{SU}(4)$.) We can consider arbitrary descendants of such chiral primaries. The $S O(6)$ content of all such protected descendants can be found for example in [2, Table 1] (the table lists the protected spectrum of supergravity on $A d S_{4} \times \mathbb{C P}^{3}$, which coincides with the spectrum of protected operators in the ABJM theory).

In any case, none of these protected operators of dimension 3 are $\mathrm{SO}(6)$ singlets. Hence our assumption (3.6) was justified.

Now that we know that there is a CFT in the space of theories $\operatorname{QFT}_{\mathcal{N}=0}\left(k_{1}, k_{2}\right)$, we can also ask whether it is attractive. For this, we need to know whether there are any relevant operators among the $O$ that we allowed in the action (the ones which are $\mathrm{SO}(6)$ singlets). This can be answered by looking for relevant operators in the ABJM theory, since our new CFT is a small deformation of it. This can in turn be analyzed using AdS/CFT: relevant operators should correspond to tachyonic operators. We can now check what these tachyonic operators are from the same table [2, Table 1]. In that table, we can see that the only scalars with negative mass squared 3 are contained in the rows denoted by $0^{+(1)}$ and $0^{-(1)}$, respectively for $p=0,1$ (dual to operators of dimension $\Delta=1,2$ ) and $p=0$ (dual to operators of dimension $\Delta=2$ ). The cases $p=2$ in $0^{+(1)}$ and $p=1$ in $0^{-(1)}$ are massless fields, dual to marginal operators. None of these operators is a singlet. Hence, there are no relevant operators which are singlets of $\mathrm{SO}(6)$ and neutral under the $\mathrm{U}(1)$ of M-theory.

To summarize, we have concluded that there exists a conformal field theory $\mathrm{CFT}_{\mathcal{N}=0}\left(k_{1}, k_{2}\right)$, with $\mathcal{N}=0$ and $\mathrm{SO}(6)$ symmetry, and that this CFT is attractive in the space $\mathrm{QFT}_{\mathcal{N}=0}\left(k_{1}, k_{2}\right)$ of field theories with this symmetry and these Chern-Simons levels.

The analysis so far ignored multi-trace interactions. We will discuss their role in section 3.7.

\footnotetext{
${ }^{3}$ The mass conventions used in [2] are given in units of $1 / R^{2}$, and after subtracting a term to make the massless case conformally invariant [13, 3.2.22]: the relation with the more usual definition is $M_{\text {here }}^{2}=$ $\frac{1}{R^{2}}\left(\frac{1}{4}\left(\operatorname{mass}_{N P}\right)^{2}-2\right)$. The formula for conformal dimensions is then the usual $\Delta(\Delta-3)=M_{\text {here }}^{2}$.
} 


\section{$3.2 \mathcal{N}=1$}

Another possibility is trying to impose $\mathcal{N}=1$ supersymmetry. The ABJM Lagrangian can be written in terms of $\mathcal{N}=1$ superfields as

$$
\begin{aligned}
& S_{\mathrm{ABJM}}=\frac{k}{4 \pi}\left(S_{\mathrm{CS}, \mathcal{N}=1}\left(\mathcal{A}_{1}\right)-S_{\mathrm{CS}, \mathcal{N}=1}\left(\mathcal{A}_{2}\right)\right)+ \\
& \int d^{2} \theta \operatorname{Tr}\left(D_{a} X_{I}^{\dagger} D^{a} X^{I}+\frac{2 \pi}{k}\left(X_{I}^{\dagger} X^{I} X_{J}^{\dagger} X_{J}-X_{I}^{\dagger} X^{J} X_{J}^{\dagger} X^{I}-2 \omega^{I K} \omega_{J L} X_{I}^{\dagger} X^{J} X_{K}^{\dagger} X_{L}\right)\right) .
\end{aligned}
$$

Here $X_{I}$ are now $\mathcal{N}=1$ superfields; we keep the same notation as for the bosonic fields in the previous subsection. The covariant derivatives are defined using the vector superfields $\mathcal{A}_{i}$. The last three terms are the $\mathcal{N}=1$ superpotential (which is a real function of the fields). It contains the symplectic matrix $\omega$, defined as being $\left(\begin{array}{ll}\epsilon & 0 \\ 0 & \epsilon\end{array}\right)$, with $\epsilon$ the antisymmetric $2 \times 2$ matrix. Notice that (3.7) makes explicit the $\operatorname{Sp}(2)$ invariance of the theory.

This time we define $\mathrm{QFT}_{\mathcal{N}=1}\left(k_{1}, k_{2}\right)$ by letting the two levels in (3.7) be unequal, and by letting the coefficients of the operators vary:

$$
\begin{aligned}
& S_{\mathrm{QFT}_{\mathcal{N}=1}\left(k_{1}, k_{2}\right)}=\frac{k_{1}}{4 \pi} S_{\mathrm{CS}, \mathcal{N}=1}\left(\mathcal{A}_{1}\right)+\frac{k_{2}}{4 \pi} S_{\mathrm{CS}, \mathcal{N}=1}\left(\mathcal{A}_{2}\right)+ \\
& \int d^{2} \theta \operatorname{Tr}\left(D_{a} X_{I}^{\dagger} D^{a} X_{I}+\left(c_{1} X_{I}^{\dagger} X^{I} X_{J}^{\dagger} X_{J}+c_{2} X_{I}^{\dagger} X^{J} X_{J}^{\dagger} X^{I}+c_{3} \omega^{I K} \omega_{J L} X_{I}^{\dagger} X^{J} X_{K}^{\dagger} X_{L}\right)\right) .
\end{aligned}
$$

This is the most general $\mathcal{N}=1$ action with levels $k_{1} \neq-k_{2}$ and $\mathrm{SO}(5)=\operatorname{Sp}(2)$ symmetry.

To establish whether there is a CFT in this space of theories, we can argue just like in the previous section. Once again we have to worry about the possible presence of marginal protected operators. Now we need to consider operators of dimension 2 integrated over the $\mathcal{N}=1$ superspace. We can consult the usual table [2, Table 1] and decompose the $\mathrm{SO}(6)$ representations under $\mathrm{Sp}(2)$. None of the representations of dimension 2 or smaller contains an $\operatorname{Sp}(2)$ singlet.

Hence, we can conclude that, in the limit (3.5), there is a CFT with Chern-Simons levels $k_{1} \neq-k_{2}$, with $\mathcal{N}=1$ supersymmetry and $\mathrm{SO}(5)$ global symmetry; and that it is an attractive fixed point in the space of theories with the same symmetries. 


\section{$3.3 \mathcal{N}=2$}

The ABJM theory can be written using $\mathcal{N}=2$ "chiral" superfields $A_{i}, B_{i}$ and vector superfields $V_{1}, V_{2}$. The action then reads

$$
\begin{aligned}
S_{\mathrm{ABJM}} & =\frac{k}{4 \pi}\left(S_{\mathrm{CS}, \mathcal{N}=2}\left(V_{1}\right)-S_{\mathrm{CS}, \mathcal{N}=2}\left(V_{2}\right)\right)+\int d^{4} \theta \operatorname{Tr}\left(e^{-V_{1}} A_{i}^{\dagger} e^{V_{2}} A_{i}+e^{-V_{1}} B_{i} e^{V_{2}} B_{i}^{\dagger}\right) \\
+ & \left(\frac{2 \pi}{k} \int d^{2} \theta \epsilon^{i j} \epsilon^{k l} A_{i} B_{k} A_{j} B_{l}+\text { c. c. }\right)
\end{aligned}
$$

where $d^{2} \theta \equiv d^{2}\left(\theta^{1}+i \theta^{2}\right)$, and the last line is the $\mathcal{N}=2$ superpotential (which is a holomorphic function of the $\mathcal{N}=2$ chiral superfields).

Once again we modify the levels, but this time in an $\mathcal{N}=2$ sense. We also want to keep the $\mathrm{SO}(4)$ invariance which is manifest in (3.9) . This leads us to

$$
\begin{aligned}
& \left.S_{\mathrm{QFT}_{\mathcal{N}=2}\left(k_{1}, k_{2}\right)}=\frac{k_{1}}{4 \pi} S_{\mathrm{CS}, \mathcal{N}=2}\left(V_{1}\right)+\frac{k_{2}}{4 \pi} S_{\mathrm{CS}, \mathcal{N}=2}\left(V_{2}\right)\right)+\int d^{4} \theta \operatorname{Tr}\left(e^{-V_{1}} A_{i}^{\dagger} e^{V_{2}} A_{i}+e^{-V_{1}} B_{i} e^{V_{2}} B_{i}^{\dagger}\right) \\
& \quad+\left(c \int d^{2} \theta \epsilon^{i j} \epsilon^{k l} A_{i} B_{k} A_{j} B_{l}+\text { c. c. }\right)
\end{aligned}
$$

This time, there is no other superpotential we can write, and all we can allow to vary is the coefficient $c$ in the second line of (3.10). At weak coupling, we can actually use the logic in [14], that goes as follows. The beta function for the coefficient $c$ should be positive for $c \gg 0$, since in that limit the Chern-Simons action is small with respect to the action of the scalars, and theory is essentially a Wess-Zumino model. At $c \ll 0$, on the other hand, one can compute the beta function for $c$ by computing the $\mathrm{R}$-charges of the fields in the theory without a superpotential. The result is that the beta function is negative. Hence, in the IR, the coupling $c$ grows when it is small, and decreases when it is large. This implies that there is a critical value in between.

At strong coupling, we could once again argue that for sufficiently small $\lambda_{+}$there will be a fixed point for a value of $c$ close to the $\mathcal{N}=6$ value. As in subsections 3.1 and 3.2 we can also check for the existence of marginal or relevant $S O(4)$ invariant chiral primaries in the spectrum. Their absence guarantees that the fixed point is attractive.

\section{$3.4 \mathcal{N}=3$}

This is case is different from all the others, in that one can actually write down the explicit Lagrangian: it is the standard $\mathcal{N}=3$ theory with the assigned Chern-Simons levels and 
field content. Using again $\mathcal{N}=2$ superfields 4 :

$$
\begin{aligned}
& \left.S_{\mathrm{CFT}_{\mathcal{N}=3}\left(k_{1}, k_{2}\right)}=\frac{k_{1}}{4 \pi} S_{\mathrm{CS}, \mathcal{N}=2}\left(V_{1}\right)+\frac{k_{2}}{4 \pi} S_{\mathrm{CS}, \mathcal{N}=2}\left(V_{2}\right)\right)+\int d^{4} \theta \operatorname{Tr}\left(e^{-V_{1}} A_{i}^{\dagger} e^{V_{2}} A_{i}+e^{-V_{1}} B_{i} e^{V_{2}} B_{i}^{\dagger}\right) \\
& \quad+2 \pi \int d^{2} \theta\left(\frac{1}{k_{1}} \operatorname{Tr}\left(B_{i} A_{i}\right)^{2}+\frac{1}{k_{2}} \operatorname{Tr}\left(A_{i} B_{i}\right)^{2}\right)
\end{aligned}
$$

In this case, the global symmetry is made up of the $\mathrm{SO}(3)_{R}$, and of the $\mathrm{SO}(3)$ that rotates the $A_{i}$ and $B_{i}$ simultaneously.

\subsection{Relations between different fixed points}

Besides the four special fixed points which we analyzed in the previous four subsections, there are other possibly interesting theories which deform the $\mathcal{N}=6$ theory in less symmetric directions.

As an example, consider the most general $\mathcal{N}=2$ single trace deformation with a Pilch-Warner type $S U(2)$ invariant superpotential [16-19]

$$
W=c_{1} \operatorname{Tr}\left(B_{i} A_{i}\right)^{2}+c_{2} \operatorname{Tr}\left(A_{i} B_{i}\right)^{2}
$$

This family of superpotentials includes both the $\mathcal{N}=3$ theory and the $\mathrm{SO}(4)$ invariant $\mathcal{N}=2$ theory $\left(c_{1}=-c_{2}=c\right)$. What is the form of the RG flow in this two-dimensional space?

Consider the chiral ring for each choice of $c_{i}$. The relations in the chiral ring $\partial W=0$ eliminate a single linear combination $W$ of the two operators $\operatorname{Tr}\left(B_{i} A_{i}\right)^{2}$ and $\operatorname{Tr}\left(A_{i} B_{i}\right)^{2}$. Hence there is a single dimension 2 chiral operator and a corresponding marginal direction for each value of the $c_{i}$. This indicates the existence of an exactly marginal line in the space of couplings which passes through the $\mathcal{N}=3$ theory and the $\mathrm{SO}(4)$ invariant $\mathcal{N}=2$ theory, and presumably connects them.

An alternative argument is that the condition for conformal invariance of the superpotential is $2 \gamma_{A_{i}}+2 \gamma_{B_{i}}=0$, a single equation in the two variables $c_{i}$.

As for the four-dimensional theory with the same superpotential [16-19], one may generalize this to superpotentials which break completely the continuous flavor symmetry, but preserves enough discrete symmetry that the number of constraints from conformal symmetry is lower than the number of coefficients in the superpotential.

Examining the spectrum of protected operators in [2, Table 1] we find another interesting fact: there is a single dimension 3 operator $(p=1)$ which is $S p(2)$ invariant,

\footnotetext{
${ }^{4}$ One could alternatively use $\mathcal{N}=3$ superfields; the ABJM action was written in this form in [15].
} 
from the $(0,2,0)$ representation of $S O(6)$. This operator could be added to the $\mathcal{N}=0$ Lagrangian to break the flavor symmetry to $S p(2)$, or to the $\mathcal{N}=1$ Lagrangian to break SUSY but preserve the $S p(2)$ flavor symmetry. This operator will have a small nonzero anomalous dimension in either theories, controlled by the small parameter $\lambda_{1}+\lambda_{2}$, and it is a good candidate to parameterize a slow flow between the $\mathcal{N}=0$ and the $\mathcal{N}=1$ fixed points.

\subsection{Other quivers}

The logic in section 2 can be applied to other $\mathrm{AdS}_{4} / \mathrm{CFT}_{3}$ duals. There are many $\mathrm{AdS}_{4}$ of the Freund-Rubin type, and one would expect that all of them should correspond to Chern-Simons-matter theories, with quivers obtained in various ways. The procedure to obtain these theories is not yet as well understood as its counterpart for four-dimensional theories. Nevertheless, there is now an infinite series of $\mathcal{N}=3$ examples [20, 21], which can be derived using the same duality procedure as in [1]. Interestingly, proposals have been made for $\mathcal{N}=2[22[25]$, and $\mathcal{N}=1$ duals [26].

In particular, we can repeat the reasoning using brane probes for the $\mathcal{N}=3$ examples (for which one can write an explicit Lagrangian). In that case, the quivers are "necklaces" consisting of $N_{\text {nodes }}$ nodes, the $i$-th being connected to the $(i+1)$-th and $(i-1)$-th by arrows going both ways (for details see [20,21]). The levels should satisfy

$$
\sum_{i=1}^{N_{\text {nodes }}} k_{i}=0 .
$$

So far for the original duals, which are of the Freund-Rubin type, and thus in particular do not have any Romans mass $F_{0}$. If one wants now to introduce a $F_{0} \neq 0$, along similar lines as in section 2 one arrives at the identification

$$
F_{0}=\sum_{i=1}^{N_{\text {nodes }}} k_{i} .
$$

One can then apply the logic in this section, to see whether these theories exist. The easiest theory to find is again the $\mathcal{N}=3$ one. In field theory, there is no particular reason to impose (3.13); that equation is valid for the theories whose gravity dual is known. (3.14) predicts that the more general theories without the relation (3.13) will have a dual with non-zero Romans mass. These solutions are not known yet, just as the dual to the $\mathcal{N}=3$ theory discussed in section 3.4 (as we will see in section 4.4). 
We can also try to generate superconformal points with different symmetries, along the lines of sections 3.1, 3.2 and 3.3. Indeed if the Chern Simons levels are alternating $k_{i}=(-1)^{i} k$, the necklace quivers enjoy $\mathcal{N}=4$ supersymmetry [12, 27], with an $S O(4)$ R-symmetry. We could then modify the levels slightly and consider a space of $\mathcal{N}=0$ theories which preserve the $S O(4)$ flavor symmetry, of $\mathcal{N}=1$ theories which preserve $S O(3)$ flavor symmetry, or $\mathcal{N}=2$ theories with $S O(2)_{R} \times S O(2)$ symmetry. There will also be other exactly conformal $\mathcal{N}=2$ theory with more general superpotentials. It would be interesting to develop this point further.

\subsection{Multitrace deformations}

In this section we would like to study the effect of multitrace terms on the existence of the four deformations of the ABJM CFT.

One could be concerned with the possibility that the existence of marginal protected multi-trace operators will destroy the perturbative construction of fixed points for the beta functions.

$$
\beta_{O}=\left(\lambda_{1}+\lambda_{2}\right) \delta \beta_{O}+\delta c_{O} \gamma_{O}
$$

If the operator is marginal in the ABJM theory, $\gamma_{O}$ will also be small, proportional to $\lambda_{1}+\lambda_{2}$. On the other hand, the planar expansion of the gauge theory gives us a hand: $\delta \beta_{O}$ will contain extra factors of $1 / N^{2}$, and $\delta c_{O}$ will still be small at a fixed point.

Such marginal operators definitely exist. $\operatorname{Tr} X^{I} \bar{X}_{J} \operatorname{Tr} X^{J} \bar{X}_{I}$ and $\operatorname{Tr} X^{I} \bar{X}_{J} \operatorname{Tr} X^{K} \bar{X}_{T} \omega_{I K} \omega^{J T}$ are protected dimension 2 double trace operators in the ABJM theory which preserve the $S p(2)$ flavor symmetry of the $\mathcal{N}=1$ theory. It is also possible to write several double trace Yukawa couplings and double or triple trace potentials for the $\mathcal{N}=0$ theory which are protected in the ABJM theory.

$\operatorname{Tr} A^{i} B_{u} \operatorname{Tr} A^{j} B_{v} \epsilon_{i j} \epsilon^{u v}$ is a chiral $S O(4)$ invariant operator in in the $\mathcal{N}=2$ theory. Although perturbatively the superpotential is not renormalized, one may imagine that non-perturbative effects in $\lambda_{-}$may introduce it. Notice that this operator is protected in the $\mathcal{N}=2$ Lagrangian as well, not just in the ABJM theory. This means that $\delta c_{O}$ will enter the $\beta$ function quadratically, instead of linearly as in (3.15), possibly leading to $\delta c_{O}$ of order $1 / N$.

Finally, there is one relevant double trace operator in the $\mathcal{N}=0$ theory, $\operatorname{Tr} X^{I} \bar{X}_{J} \operatorname{Tr} X^{J} \bar{X}_{I}$. 


\section{Gravity duals}

The conformal field theories we have described in section 3 should all have gravity duals. We were able to find duals only for the $\mathcal{N}=0$ and $\mathcal{N}=1$ cases, which ironically are the ones with less control from the field theory side.

Our electric basis will be made of the internal fluxes, that is, the ones with no indices in the spacetime, just as in [28]. These include a zero-form $F_{0}$ (the Romans mass), a two-form $F_{2}$, a four-form $F_{4}$, a volume form $F_{6}$.

\section{1 $\mathcal{N}=0$}

In this subsection 5 , we will look for $\mathcal{N}=0$ solutions with $\mathrm{SO}(6)$ symmetry. This means that we can just take the metric to be the usual Fubini-Study metric on $\mathbb{C P}^{3}$.

We will also assume that the dilaton is constant, as well as the warping (the function of the internal coordinates multiplying the AdS metric). Actually, when the latter is constant, it can be just set to zero by rescaling. The equations of motion for the dilaton and metric are then (in the string frame)

$$
R_{10}=\frac{1}{2} H^{2}, \quad R_{M N}-\frac{1}{2} g_{M N} R_{10}=g^{2} T_{M N}
$$

with

$$
\begin{aligned}
T_{M N}= & \sum_{k=0}^{3} \frac{1}{2}\left(\frac{1}{(2 k-1) !} F_{M P_{1} \ldots P_{2 k-1}} F_{N} P_{1} \ldots P_{2 k-1}-\frac{1}{2(2 k) !} g_{M N} F_{P_{1} \ldots P_{2 k}} F^{P_{1} \ldots P_{2 k}}\right)+ \\
& \frac{1}{2 g_{s}^{2}}\left(\frac{1}{2} H_{M P Q} H_{N} P Q-\frac{1}{12} g_{M N} F_{P Q R} F^{P Q R}\right) .
\end{aligned}
$$

For a geometry $\mathrm{AdS}_{4} \times M_{6}$ with $M_{6}$ Einstein, the whole content of Einstein's equations is in their four-dimensional trace

$$
R_{4}=-g_{s}^{2} \sum_{k} F_{k}^{2}
$$

and their six-dimensional trace

$$
R_{6}=\frac{1}{2}\left(3 H^{2}+g_{s}^{2} \sum_{k}(k-3) F_{k}^{2}\right),
$$

\footnotetext{
${ }^{5}$ Some of the computations in this section were done, in a different context, in conversations with Mariana Graña. The idea of finding solutions by switching on only singlets of the internal SU(3) structure, as we will do below, is of course not new; in massive IIA, see [29. for non-supersymmetric examples, 30. for supersymmetric ones, and even [31] for early examples.
} 
where, again, the F's are internal. The equations of motion are, then, these two and the one for the dilaton (the first in (4.1) ). In fact, (4.3) just sets the value for the fourdimensional cosmological constant. It is also convenient to combine the first in (4.1) with the sum of (4.3) and (4.4), to obtain

$$
2 H^{2}=g_{s}^{2} \sum_{k}(5-k) F_{k}^{2}
$$

We also have to consider the equations of motion for the internal fluxes $H$ and $F_{k}$, and their Bianchi identity. If we assume that no sources are present, these read

$$
d F=H \wedge F, \quad d * F=-H \wedge F, \quad d H=0, \quad d * H=-g_{s}^{2} \sum_{k=0}^{2}\left(F_{2 k} \wedge * F_{2 k+2}\right) .
$$

So far, we have only used that the internal space is Einstein. We now start using the fact that it is also Kähler. We will consider an Ansatz for the fluxes

$$
F=f_{0}, \quad F_{2}=f_{2} J_{0}, \quad F_{4}=f_{4} \frac{J_{0}^{2}}{2}, \quad F_{6}=f_{6} \frac{J_{0}^{3}}{6}, \quad H=0,
$$

where $J_{0}$ is the Kähler form and the $f_{i}$ are taken to be constant. The motivation for this Ansatz is simplicity: the idea is to use nothing but singlets of $\mathrm{U}(3)$ (the structure group of the manifold). In the supersymmetric case, a similar idea was used in [30]; in the non-supersymmetric case, in [29].

Let us now see whether we can find any solutions with this Ansatz. The equations of motion and Bianchi identities for the internal fluxes are (4.6). The first three of them are trivially satisfied, because $H=0$ and $J_{0}$ is closed. To evaluate the fourth, we use

$$
F \cdot F \mathrm{vol}=\frac{1}{k !} F_{m_{1} \ldots m_{k}} F^{m_{1} \ldots m_{k}} \operatorname{vol}=(-)^{k} F \wedge * F
$$

to compute

$$
1 \cdot 1=\frac{J_{0}^{3}}{6 !} \cdot \frac{J_{0}^{3}}{6 !}=1, \quad J_{0} \cdot J_{0}=\frac{J_{0}^{2}}{2} \cdot \frac{J_{0}^{2}}{2}=3 .
$$

Then the fourth in (4.6) gives

$$
f_{0} f_{2}+2 f_{2} f_{4}+f_{4} f_{6}=0
$$

We now look at the equations of motion of the dilaton and metric. For the internal metric, we use the conventions of section 4.2, namely, we take the metric in (4.23), restricted to the case $\sigma=2$. This gives $R_{6}=\frac{48}{R^{2}}$. Using (4.7) and (4.9), (4.3), (4.4) and 
(4.5) read respectively

$$
\begin{aligned}
& \Lambda=-\frac{12}{R^{2}} \\
& \frac{48}{R^{2}}=\frac{3}{2} g_{s}^{2}\left(-f_{0}^{2}-f_{2}^{2}+f_{4}^{2}+f_{6}^{2}\right) \\
& 5 f_{0}^{2}+9 f_{2}^{2}+3 f_{4}^{2}-f_{6}^{2}=0 .
\end{aligned}
$$

(4.11) comes simply from $R_{4}=4 \Lambda$ and $R_{10}=0$ (which is the equation of motion for the dilaton, the first in (4.1), for $H=0$ ). Equations (4.11) and (4.12) determine $\Lambda$ and $g_{s}$ in terms of the flux parameters $f_{2 k}$. The latter are constrained, however, by (4.10) and (4.13). One can solve this system by writing 6

$$
f_{0}^{2}\left(f_{2}^{2}+5\left(2 f_{2}+f_{6}\right)^{2}\right)=\left(f_{6}^{2}-9 f_{2}^{2}\right)\left(2 f_{6}+f_{2}\right)^{2}
$$

in the sense that one can use this equation to determine $f_{0}$ in terms of $f_{2}$ and $f_{6}$.

Notice that for the $\mathcal{N}=6$ solution, $f_{6}^{2}=36 /\left(g_{s}^{2} R^{2}\right)$ and $f_{2}^{2}=12 /\left(g_{s}^{2} R^{2}\right)$, whereas $f_{0}=f_{4}=0$.

At this point, as far supergravity goes, we have found a family of solutions, parameterized by $f_{2}, f_{6}$, and by $R$, which at the moment is a free parameter. There is also the possibility of adding a closed $B$ field, which can be parameterized by the number

$$
b \equiv \int_{\mathbb{C P}^{1}} B .
$$

We now want to show that this family of solutions survive flux quantization and stringy corrections.

\subsubsection{Flux quantization}

As we already remarked in section 2, the fluxes that couple to the brane worldsheets are defined as $\tilde{F}_{k} \equiv\left(e^{-B} F\right)_{k}$; for example, $\tilde{F}_{2} \equiv F_{2}-B F_{0}$. The quantization law then reads

$$
\mathbb{Z} \ni n_{2 k}=\int_{\mathbb{C P} k} \tilde{F}_{2 k} .
$$

If we use the Ansatz (4.7), we get that

$$
f_{k}=\frac{n_{k}^{b}}{R^{k} v_{k}}
$$

\footnotetext{
${ }^{6}$ One can divide both equations by $f_{6}$, then use (4.10) to express $f_{4} / f_{6}$ in terms of $f_{2} / f_{6}$ and $f_{0} / f_{6}$, then plug this into (4.13) and solve for $f_{0} / f_{6}$.
} 
where $v_{k}$ are some numerical coefficients (the volumes of the $\mathbb{C P}^{k / 2}$ for $R=1$ ), the $n_{k}^{b}$ are defined as

$$
\left(\begin{array}{c}
n_{0}^{b} \\
n_{2}^{b} \\
n_{4}^{b} \\
n_{6}^{b}
\end{array}\right) \equiv\left(\begin{array}{cccc}
1 & 0 & 0 & 0 \\
b & 1 & 0 & 0 \\
\frac{1}{2} b^{2} & b & 1 & 0 \\
\frac{1}{6} b^{3} & \frac{1}{2} b^{2} & b & 1
\end{array}\right)\left(\begin{array}{c}
n_{0} \\
n_{2} \\
n_{4} \\
n_{6}
\end{array}\right),
$$

and $b$ was defined in (4.15). Notice that in section 2, we called $n_{2}=k$ and $n_{6}=N$.

Hence, for each choice $\left\{n_{k}\right\}$ of the four flux integers, flux quantization gives us the four equations (4.17). As we remarked after (4.14), the family of solutions we had before imposing flux quantization has four parameters: $f_{2}, f_{6}, R$ and $b$. This sounds promising, but in general such a system of real equations might or might not have solutions, depending on the coefficients and hence on the $n_{k}$. But we do know that the system has a solution, namely the $\mathcal{N}=6$ solution [2,3]. Actually, the solutions we care about in this paper are the ones which are small perturbations of this $\mathcal{N}=6$ solutions. Let us think of (4.17) as a map from $\left(f_{2}, f_{6}, R, b\right)$ to $\left(n_{0}, n_{2}, n_{4}, n_{6}\right)$. All we need to do, then, is to make sure that the image of this map extends in the $n_{0}$ direction around the $\mathcal{N}=6$ solution. This can be done explicitly in perturbation theory. If one just includes a small $n_{0} \ll n_{2}, n_{6}$, without including $n_{4}$ for simplicity, one finds $\delta f_{0}=n_{0}, \delta f_{4}=-\frac{1}{5} \delta f_{0}, \delta b=\frac{R^{4}}{n_{2}} \delta f_{4}$. This shows that the $\mathcal{N}=6$ solution is not isolated in the $n_{0}$ direction. We have also found solutions of this type numerically.

Stringy corrections are also under control. The solutions to these equations will be analytic in the coefficients, if one lets them vary continuously. So, once again, if one starts from the $\mathcal{N}=6$ solution, for which $R$ can be made large and $g_{s}$ small, and one adds some small amount of $n_{0}, R$ will be still large, and $g_{s}$ will still be small.

Finally, one should wonder whether this solution is stable, since we no longer have supersymmetry to protect it. Fortunately, the $\mathcal{N}=6$ solution is not only stable (as it should be because of supersymmetry): its mass spectrum has also a gap above the Breitenlohner-Freedman bound, as one can again see from [2, Table 1]. In other words, not only do all the fields in the spectrum satisfy the bound: none saturates it. For $\mathcal{N}=0$ solutions that are small perturbations of the $\mathcal{N}=6$ solution, which are the ones we are interested in, the Breitenlohner-Freedman will then still be satisfied, since no mass can suddenly fall below the bound.

In conclusion, we have found a set of solutions of IIA with isometry group $\mathrm{SU}(4)=\mathrm{SO}(6)$, with no preserved supersymmetry, and with non-zero Romans mass $F_{0}$. Using the logic 
in section 2, we propose that these solutions should be dual to the theories discussed in subsection 3.1, with the map

$$
k_{1}=n_{2}+n_{0}, \quad k_{2}=-n_{2}, \quad N_{1}=n_{6}+n_{4}, \quad N_{2}=n_{6} .
$$

at least when $F_{0}$ is small with respect to the other flux quanta.7

\subsubsection{Probe branes}

In this section, we perform a probe brane analysis on the $\mathcal{N}=0$ vacua we just found.

Consider a D2 extended along the three non-radial directions of spacetime. We want to ask whether such a brane is stable or unstable to expanding in the radial direction. This will tell us whether the potential of the dual theory is positive definite or not.

The potential for such a D2 is

$$
V=-\mu_{2}\left[\int d^{3} \sigma \sqrt{-|g|}+\int C_{3}\right] .
$$

Here, $\mu_{2}$ is the D2 tension. $|g|$ is the determinant of the (pull-back of) the spacetime metric; the relevant part is the standard $\mathrm{AdS}_{4}$ metric $\frac{R_{A d S}^{2}}{r^{2}}\left(d r^{2}+\left(d x^{0}\right)^{2}+\left(d x^{1}\right)^{2}+\left(d x^{2}\right)^{2}\right)$. $C_{3}$ is the three-form potential. When we described the $\mathcal{N}=0$ vacua earlier in this section, recall that we chose the internal fluxes as our electric basis. Among which we had $F_{6}=f_{6} \operatorname{vol}_{6}$. So far we have not used any of the "magnetic" fluxes $\tilde{F}_{i}$, namely the ones which have also legs along the spacetime. These can be obtained from the "electric" internal fluxes by duality; in particular, we have $\tilde{F}_{4}=f_{6} \mathrm{vol}_{\text {AdS }}$. Taking a potential $C_{3}$ with no leg in the radial direction, we have $d C_{3}=d r \wedge \partial_{r} C=\tilde{F}_{4}$, which gives us

$$
C_{3}=-\frac{R_{\mathrm{AdS}}^{4}}{3 r^{3}} f_{6} d x^{0} \wedge d x^{1} \wedge d x^{2}
$$

Summing up, we have

$$
V=-\frac{\mu_{2}}{r^{3}}\left(\frac{R_{\mathrm{AdS}}^{3}}{g_{s}}-\frac{f_{6}}{3} R_{\mathrm{AdS}}^{4}\right)=-\mu_{2} \frac{R_{\mathrm{AdS}}^{3}}{r^{3}}\left(\frac{1}{g_{s}}-\frac{R}{6} f_{6}\right)
$$

where we have used $R_{\mathrm{AdS}}=\sqrt{-\frac{3}{\Lambda}}$, and (4.11).

\footnotetext{
${ }^{7}$ The reader might be unhappy about the seeming asymmetry of this formula. Notice, however, that a shift of $B$ by the closed form with $2 \pi$ period will send $\tilde{F}_{6} \rightarrow \tilde{F}_{6}+\tilde{F}_{4}$, which (via (4.16) ) will exchange the role of $N_{1}$ and $N_{2}$ in (4.19). At the same time, this will also exchange the role of $k_{1}$ and $k_{2}$. Naively this would also generate contributions of order $k$ to $N_{1}$ and $N_{2}$; presumably the resolution to the puzzle comes from the Riemann ${ }^{2}$ terms in the brane action, which indeed should give rise to corrections of order $\left(l_{s} / R\right)^{4} \sim k / N$. It would be interesting to check this in detail.
} 
Notice that, as remarked earlier in this section, for the $\mathcal{N}=6$ solution we have $f_{6}=\frac{6}{g_{s} R}$; hence, in that case, the potential in (4.22) is identically zero. This is how it should be: the potential for the $\mathcal{N}=6 \mathrm{CFT}$ has flat directions that correspond to the D2 moving along $r$. In the general case, one can just use (4.12), (4.14) and (4.10) to express $V$ in (4.22) as a (complicated) function of $R, f_{2}$ and $f_{6}$. Actually $R$ is just a multiplicative overall factor; one can also factor out $f_{2}$ and study the remaining function of $\frac{f_{6}}{f_{2}}$. Upon noticing from (4.14) that $\left|\frac{f_{6}}{f_{2}}\right| \geq 3$, one finds (if $f_{6}$ and $f_{2}$ have equal sign) that $V \leq 0$ : the electric repulsion term wins over the gravitational attractive term. These $\mathcal{N}=0$ vacua are then non-perturbatively unstable towards nucleation of D2 branes. The dual field theories will then have an unstable potential.

\section{$4.2 \mathcal{N}=1$}

In this case, field theory instructs us to look for a family of solutions on $\mathbb{C P}^{3}$ with $\mathcal{N}=1$ supersymmetry, SO(5) symmetry, and non-zero Romans mass.

In fact, such a family already exists [5]. The metric is no longer the Fubini-Study metric. Topologically, $\mathbb{C P}^{3}$ is an $S^{2}$ fibration over $S^{4}$. The metric can be written as

$$
d s_{6}^{2}=R^{2}\left(\frac{1}{8}\left(d x^{i}+\epsilon^{i j k} A^{j} x^{k}\right)^{2}+\frac{1}{2 \sigma} d s_{S^{4}}^{2}\right)
$$

where $x^{i}$ are such that $\sum_{i=1}^{3}\left(x^{i}\right)^{2}=1, A^{i}$ are the components of an $\mathrm{SU}(2)$ connection on $S^{4}$ (with $p_{1}=1$ ), and $d s_{S^{4}}^{2}$ is the round metric on $S^{4}$ (with radius one). $R$ is an overall radius. For $\sigma=2$, (4.23) is the usual Fubini-Study metric, whose isometry group is $\mathrm{SO}(6)$; in this case, the metric has a coset structure $\mathrm{SU}(4) / \mathrm{U}(3)$. For $\sigma \neq 2$, the isometry group is simply the $\mathrm{SO}(5)$ of the base $S^{4}$. Actually, the metric still comes from a coset: $\mathrm{Sp}(2) / \mathrm{Sp}(1) \times \mathrm{U}(1)$. This latter fact was emphasized in [32], who redid the computations in [5] using this coset structure.

At the level of supergravity, the $\mathcal{N}=1$ solutions found in [5] are a family with four parameters: the two parameters $R$ and $\sigma$ in the metric (4.23), the string coupling $g_{s}$, and a parameter $b$ similar to the one defined in (4.15). The difference in this case is that supersymmetry requires the NS curvature $H$ be non-zero (see [5, Eq. (2.2)]). One can solve that constraint by writing

$$
B=\frac{m}{\tilde{m}} J+B_{0}
$$

where $m$ and $\tilde{m}$ are two functions of $\sigma$, and $B_{0}$ is a closed two-form. One can then define

$$
b \equiv \int_{\mathbb{C P}^{1}} B_{0} .
$$


Once again, we have a family of solutions with four parameters. The flux quantization conditions read

$$
\left(\begin{array}{c}
\frac{5}{2 r g_{s}} m_{0} \\
4 \pi \frac{r}{g_{s}} \frac{(\sigma-1)}{(\sigma+2)} \\
-\frac{4}{3} \pi^{2} \frac{r^{3}}{g_{s}} m_{0} \frac{(\sigma-1)(1+2 \sigma)}{\sigma^{2}(\sigma+2)^{2}} \\
-\frac{4}{15} \pi^{3} \frac{r^{5}}{g_{s}} \frac{(1+2 \sigma)\left(\sigma^{2}-12 \sigma-4\right)}{\sigma^{2}(\sigma+2)^{2}}
\end{array}\right)=\left(\begin{array}{c}
n_{0}^{b} \\
n_{2}^{b} \\
n_{4}^{b} \\
n_{6}^{b}
\end{array}\right)
$$

where $n_{k}^{b}$ are defined as in (4.18), and

$$
m_{0} \equiv \sqrt{(\sigma-2 / 5)(2-\sigma)}
$$

The vector on the left of (4.26) is nothing but $\left(F_{0}, \int_{\mathbb{C P}^{1}} \tilde{F}_{2}, \int_{\mathbb{C P}^{2}} \tilde{F}_{4}, \int_{\mathbb{C P}^{3}} \tilde{F}_{6}\right)$, where $\tilde{F}_{k} \equiv$ $\left(e^{-\frac{m}{\tilde{m}} J} F\right)_{k}$, just like in section 4.1.

Now we have a system given by the four equations (4.26) for the four parameters $R$, $\sigma, g_{s}$ and $b$. To show that the system has solutions close to the $\mathcal{N}=6$ solution 8 , we have proceeded using perturbation theory, introducing a small $n_{0} \ll n_{2}, n_{6}$, along the lines explained in section 4.1 for the $\mathcal{N}=0$ solutions. Once again, we have also found numerical examples.

It is very natural to say that these solutions should be dual to the theories discussed in 3.2, with the same matching of discrete parameters as in (4.19).

\section{$4.3 \mathcal{N}=2$}

In this case, we cannot offer the gravity dual to the field theories discussed in 3.3 . The reason is essentially that the amount of symmetry is smaller: in both the $\mathcal{N}=0$ and $\mathcal{N}=1$ cases, the metric was homogeneous: the orbit of the isometry group (respectively, $\mathrm{SO}(6)=\mathrm{SU}(4)$ and $\mathrm{SO}(5)=\mathrm{Sp}(2))$ was the whole space. In the $\mathcal{N}=2$ case, the isometry group is $\mathrm{SO}(4)$, and its orbits have codimension 1 . This means that, this time, one really has to solve differential equations in one variable.

Another difficulty is that the $\mathcal{N}=2$ solution we are looking for is not going to be unlike the supersymmetric solutions known so far, in a sense we now specify. Supersymmetric solutions can be broadly divided in two classes. In type IIA, there are two supersymmetry

\footnotetext{
${ }^{8}$ Several solutions to this system were found in [5]; in that paper, however, there was no reason to look for them particularly close to the $\mathcal{N}=6$ solution.
} 
parameters; for an $\mathcal{N}=1$ vacuum solution, they can be decomposed as

$$
\begin{aligned}
& \epsilon^{1}=\zeta_{+} \otimes \eta_{+}^{1}+\zeta_{-} \otimes \eta_{-}^{1} \\
& \epsilon^{2}=\zeta_{-} \otimes \eta_{+}^{2}+\zeta_{+} \otimes \eta_{-}^{2}
\end{aligned}
$$

where $\zeta_{+}$is a chiral four-dimensional spinor, $\eta_{+}^{1,2}$ are two chiral six-dimensional spinors, and $\zeta_{-}=\left(\zeta_{+}\right)^{*}, \eta_{-}^{1,2}=\left(\eta_{+}^{1,2}\right)^{*}$ to make sure $\epsilon^{1,2}$ are Majorana. If $\eta^{1}$ and $\eta^{2}$ are proportional, the solution is said to be "SU(3) structure"; if they are not, it is called $\mathrm{SU}(3) \times \mathrm{SU}(3)$ structure. These strange-sounding names come from the general classification of supersymmetric vacua using generalized complex geometry [28, 33]). The $\mathcal{N}=1$ solutions of [5], that we just reviewed in section 4.2, are $\mathrm{SU}(3)$ structure; as are, to the best of our knowledge, all known supersymmetric $\mathrm{AdS}_{4}$ solutions 9

For $\mathcal{N}=2$ solutions 10 , one needs two four-dimensional spinors, and as a consequence we need another pair of internal six-dimensional spinors too:

$$
\begin{aligned}
\epsilon^{1} & =\sum_{a=1}^{2}\left(\zeta_{+}^{a} \otimes \eta_{+}^{1, a}++\zeta_{-}^{a} \otimes \eta_{-}^{1, a}\right) \equiv \sum_{a=1}^{2} \epsilon^{1, a} \\
\epsilon^{2} & =\sum_{a=1}^{2}\left(\zeta_{+}^{a} \otimes \eta_{+}^{2, a}++\zeta_{-}^{a} \otimes \eta_{-}^{2, a}\right) \equiv \sum_{a=1}^{2} \epsilon^{1, a}
\end{aligned}
$$

In this case, the pair $\eta^{1, a=1}, \eta^{2, a=1}$ and the pair $\eta^{1, a=2}, \eta^{2, a=2}$ have each to solve the same equations as in the $\mathcal{N}=1$ case; but with the same metric and fluxes. Also, the R-symmetry generator $R$ sends the first solution into the second. That implies that $\epsilon^{i, a=1}=R_{j}^{i} \epsilon^{j, a=2}$ for some matrix $R_{j}^{i}$. Hence, the pair for $a=1$ is $\mathrm{SU}(3)$, so will be the pair for $a=2$. For this reason, it still makes sense to divide solutions into $\mathrm{SU}(3)$ and $\mathrm{SU}(3) \times \mathrm{SU}(3)$, like we $\operatorname{did}$ in the $\mathcal{N}=1$ case.

$\mathrm{SU}(3)$-structure solutions are characterized by a set of equations first found in [34]; in the present notation, they can be found in [5, Eq. $(2.2,2.6)]$. To have an $\mathcal{N}=2$ solution with non-zero $F_{0}$, one needs to find two solutions $\left(J_{1}, \Omega_{1}\right)$ and $\left(J_{2}, \Omega_{2}\right)$ to those equations; the index is the $a=1,2$ that we saw in (4.29). As we mentioned above, these two pairs should solve the equations with the same fluxes $H, F_{2 k}$ and the same metric $g$. The latter is determined as

$$
g=J_{1} I_{1}=J_{2} I_{2}
$$

\footnotetext{
${ }^{9}$ It should be easy, however, to generate new supersymmetric solutions beyond this class by acting with a solution-generating technique, as in [6].

${ }^{10}$ Here we are talking about solutions with non-vanishing RR fields. Without this assumption, the supersymmetry equations do not mix the $\epsilon^{1}$ and $\epsilon^{2}$, and it is possible to achieve $\mathcal{N}=2$ by having two different $\zeta$ 's in the two rows of (4.28).
} 
where $I_{a}$ are two almost complex structures such that $\Omega_{a}$ are their $(3,0)$ forms. Now, for $F_{0} \neq 0$, one finds from [5, Eq. (2.2)] that 11

$$
H=\frac{2}{5} g_{s} F_{0} \operatorname{Re} \Omega \text {. }
$$

This equation should be true for both $\Omega_{a}$. Hence one gets that $\operatorname{Re} \Omega_{1}=\operatorname{Re} \Omega_{2}$. But both $\Omega_{a}$ should be decomposable (namely, locally the wedge of three one-forms), because they should be $(3,0)$ forms with respect to same almost complex structures $I_{a}$. This implies that each $\operatorname{Re} \Omega_{a}$ should determine the whole of $\Omega_{a}$ (see for example [35]); hence one gets $\Omega_{1}=\Omega_{2}$. But a decomposable non-degenerate form $\Omega$ actually determines an almost complex structure $I$ under which it should be a $(3,0)$-form. This means that also $I_{1}=I_{2}$. Finally, because of (4.30), $J_{1}=J_{2}$. So the two solutions to the supersymmetry equations are actually the same.

In summary, we have shown in full generality that a supergravity solution with extended supersymmetry and $F_{0} \neq 0$ cannot be $\mathrm{SU}(3)$-structure.

This result is a complication for us: the equations for the $\mathrm{SU}(3) \times \mathrm{SU}(3)$ case are far more complicated than the ones for the $\mathrm{SU}(3)$ case. Although both $\mathrm{SU}(3)$ and $\mathrm{SU}(3) \times$ $\mathrm{SU}(3)$ are particular cases of [28, Eq. (7.1), (7.2)], written in the language of generalized complex geometry, the $\mathrm{SU}(3) \times \mathrm{SU}(3)$ case needs to be massaged significantly before they can be applied to any concrete situation. Although work is in progress on this, we do not have as yet a solution to offer.

The fact that the solution has to be $\mathrm{SU}(3) \times \mathrm{SU}(3)$ structure can also be seen purely from field theory, as we now proceed to show. From the general rules of the AdS/CFT correspondence, we expect to be able to compare domain wall D-branes in $\mathrm{AdS}_{4}$, extended along the subspace defined by $r=$ const in Poincaré coordinates, with vacua of the CFT. Also, BPS domain walls in $\mathrm{AdS}_{4}$ should correspond to supersymmetric vacua in the CFT.

For the particular CFT described in section 3.3, the gauge group is $\mathrm{U}(N) \times \mathrm{U}(N)$, and $N$ should be large for the $\alpha^{\prime}$ corrections to be under control on the gravity side; but let us imagine we are only giving expectation value to a $1 \times 1$ block. In this abelian case, the moduli space of supersymmetric vacua is given by

$$
\sum_{i=1}^{2}\left(\left|A_{i}\right|^{2}-\left|B_{i}\right|^{2}\right)=0,
$$

since the superpotential in (3.10) vanishes in this abelian case.

\footnotetext{
${ }^{11}$ This equation is also related to (4.24).
} 
One also has to take care of possible actions on the moduli space by the gauge group. The moduli space of the original $\mathrm{U}(1) \times \mathrm{U}(1)$ ABJM theory is a $\mathbb{C}^{4} / Z_{k}$ parameterized by the $A_{i}, \bar{B}_{i}$. The $A_{i}, \bar{B}_{i}$ fields all have the same charge under the linear combination $\mathcal{A}_{1}-\mathcal{A}_{2}$ of the two U(1) gauge fields, so the naive Higgsing would give as a moduli space the cone over $\mathbb{C P}^{3}$. The particular choice of opposite Chern-Simons levels replaces the naive U(1) gauging by the discrete $\mathbb{Z}_{k}$ quotient, to give $\mathbb{C}^{4} / \mathbb{Z}_{k}$.

If the sum of the levels is not zero, the $\mathrm{U}(1)$ gauging is no longer discretized, and it happens in full. The moduli space of a $\mathcal{N}=2$ theory should be Kähler, and indeed (4.32) is the correct equation for a Kähler quotient of $\mathbb{C}^{4}$.

Let us now look at the gravity side. The BPS conditions for domain walls can be written conveniently [36] in terms of the pure spinors $\Phi_{ \pm}$of generalized complex geometry, that also appear in the supersymmetry equations for the background [28]. We expect the abelian vacua in the CFT should correspond to a D2 domain wall, situated at $r=$ const and pointlike in the internal $\mathbb{C P}^{3} 12$ For such a brane, in the conventions of [28, Eq. (7.1), (7.2)] the only condition is

$$
\operatorname{Im}\left(e^{i \theta} \Phi_{+(0)}^{a}\right)_{\mid}=0
$$

where $e^{i \theta}$ is the phase of $\mu, \Phi_{+(0)}$ is the zero-form part of the $\Phi_{+}$pure spinor, $a$ is the index introduced in (4.29), and | denotes restriction to the point in $\mathbb{C P}^{3}$ where the D2 is located. Now, the crucial point is that, in the $\mathrm{SU}(3)$ case, it was shown in [28, Sec. 7] that the left hand side of (4.33) is constant. This means that (4.33) either has solution everywhere on the $\mathbb{C P}^{3}$, or nowhere. This does not match with what we found on the CFT side, namely the single equation (4.32).

In the $\mathrm{SU}(3) \times \mathrm{SU}(3)$ case, there is no reason for the left hand side of (4.33) to be constant. The equation (4.33) should then just match (4.32). Indeed, it is easy to argue from the isometries of the problem that $\Phi_{+(0)}^{1}=\Phi_{+(0)}^{2}$ is constant on the orbits of the $S O(4)$ flavor symmetry, which are given by

$$
\sum_{i=1}^{2}\left(\left|A_{i}\right|^{2}-\left|B_{i}\right|^{2}\right)=t .
$$

The field theory predicts that the 4.33 will be satisfied at $t=0$.

It remains to interpret the $\mathrm{U}(1)$ gauging from gravity. First of all, in the ABJM case, we saw that the moduli space of the field theory is $\mathbb{C}^{4} / \mathbb{Z}_{k}$. From the point of view of the D2 domain walls, the combination of the radial motion in $A d S_{4}$ and the motion in $\mathbb{C P}^{3}$

\footnotetext{
${ }^{12}$ Domain walls wrapping higher-dimensional subspaces of $\mathbb{C P}^{3}$ will presumably come from Myers-like effects in the CFT.
} 
gives a cone over $\mathbb{C P}^{3}$; an extra $\mathrm{U}(1)$ circle arises from dualization of the wordvolume gauge field, and one reproduces this way the $\mathbb{C}^{4} / \mathbb{Z}_{k}$ moduli space.

In the case with unequal levels, we saw instead that an $\mathrm{U}(1)$ gauging happens, which is complexified by the condition (4.32). From the point of view of the D2 domain wall, the $F_{0}$ background induces a Chern-Simons coupling on the worldvolume, and the worldvolume gauge field becomes massive, together with a transverse scalar, which is its $\mathcal{N}=2$ supersymmetric partner.

\section{$4.4 \mathcal{N}=3$}

This case is very similar to the $\mathcal{N}=2$ case. As for that case, we cannot offer a gravity solution. And again to our partial excuse, we can apply the gravity argument we saw in section 4.3, and conclude that the solution must be $\mathrm{SU}(3) \times \mathrm{SU}(3)$.

The comparison of D2 BPS domain walls with vacua of the CFT also goes along similar lines. The BPS equation reads again (4.33), but with $a$ going from 1 to 3 . This should correspond to the three equations for supersymmetric vacua for (3.11), that read

$$
\sum_{i=1}^{2}\left(\left|A_{i}\right|^{2}-\left|B_{i}\right|^{2}\right)=0, \quad \sum_{i=1}^{2} A_{i} B_{i}=0
$$

or, more symmetrically, $\sum_{i=1}^{2} X_{i}^{\dagger} \sigma_{\alpha} X_{i}$, with $X_{i}=\left(\begin{array}{l}A_{i} \\ B_{i}\end{array}\right)$, and $\sigma_{\alpha}$ Pauli matrices.

These are the conditions for the $\mathrm{U}(1)$ hyper-Kähler quotient of $\mathbb{C}^{4}$. In this case $\Phi_{+(0)}^{a}$ should be constant on the orbits of the $\mathrm{SO}(3)$ flavor symmetry, which are given by

$$
\sum_{i=1}^{2}\left(\left|A_{i}\right|^{2}-\left|B_{i}\right|^{2}\right)=t_{R}, \quad \sum_{i=1}^{2} A_{i} B_{i}=t_{C} .
$$

The field theory predicts that the 4.33 will be satisfied at $t_{R}=t_{C}=0$.

Acknowledgments. We would like to thank G. Moore, J. Maldacena and A. Zaffaroni for interesting discussions. D. G. is supported in part by the DOE grant DE-FG0290ER40542 and in part by the Roger Dashen membership in the Institute for Advanced Study. A. T. is supported in part by DOE grant DE-FG02-91ER4064.

\section{References}

[1] O. Aharony, O. Bergman, D. L. Jafferis, and J. Maldacena, " $\mathcal{N}=6$ superconformal Chern-Simons-matter theories, M2-branes and their gravity duals," 0806.1218. 
[2] B. E. W. Nilsson and C. N. Pope, "Hopf fibration of eleven-dimensional supergravity," Class. Quant. Grav. 1 (1984) 499.

[3] D. P. Sorokin, V. I. Tkach, and D. V. Volkov, "On the relationship between compactified vacua of $d=11$ and $d=10$ supergravities," Phys. Lett. B161 (1985) 301-306.

[4] J. H. Schwarz, "Superconformal Chern-Simons theories," JHEP 11 (2004) 078, hep-th/0411077.

[5] A. Tomasiello, "New string vacua from twistor spaces," Phys. Rev. D78 (2008) 046007, 0712.1396.

[6] O. Lunin and J. M. Maldacena, "Deforming field theories with $\mathrm{U}(1) \times \mathrm{U}(1)$ global symmetry and their gravity duals," JHEP 05 (2005) 033, hep-th/0502086.

[7] E. Imeroni, "On deformed gauge theories and their string/M-theory duals," JHEP 10 (2008) 026, 0808.1271.

[8] E. Witten, "Baryons and branes in anti de Sitter space," JHEP 07 (1998) 006, hep-th/9805112.

[9] G. W. Moore and N. Seiberg, "Taming the Conformal Zoo," Phys. Lett. B220 (1989) 422.

[10] O. Aharony, O. Bergman, and D. L. Jafferis, "Fractional M2-branes," 0807.4924.

[11] S. Mukhi and C. Papageorgakis, "M2 to D2," JHEP 05 (2008) 085, 0803.3218.

[12] M. Benna, I. Klebanov, T. Klose, and M. Smedback, "Superconformal Chern-Simons Theories and $\mathrm{AdS}_{4} / \mathrm{CFT}_{3}$ Correspondence," 0806.1519.

[13] M. J. Duff, B. E. W. Nilsson, and C. N. Pope, "Kaluza-Klein supergravity," Phys. Rept. 130 (1986) 1-142.

[14] D. Gaiotto and X. Yin, "Notes on superconformal Chern-Simons-matter theories," JHEP 08 (2007) 056, arXiv:0704.3740 [hep-th].

[15] I. L. Buchbinder et al., "ABJM models in $\mathcal{N}=3$ harmonic superspace," JHEP 03 (2009) 096, 0811.4774.

[16] R. Corrado, M. Gunaydin, N. P. Warner, and M. Zagermann, "Orbifolds and flows from gauged supergravity," Phys. Rev. D65 (2002) 125024, hep-th/0203057. 
[17] R. Corrado and N. Halmagyi, " $\mathcal{N}=1$ Field Theories and Fluxes in IIB String Theory," Phys. Rev. D71 (2005) 046001, hep-th/0401141.

[18] S. Benvenuti and A. Hanany, "Conformal manifolds for the conifold and other toric field theories," JHEP 08 (2005) 024, hep-th/0502043.

[19] N. Halmagyi, K. Pilch, C. Romelsberger, and N. P. Warner, "Holographic duals of a family of $\mathcal{N}=1$ fixed points," JHEP 08 (2006) 083, hep-th/0506206.

[20] D. L. Jafferis and A. Tomasiello, "A simple class of $\mathcal{N}=3$ gauge/gravity duals," JHEP 10 (2008) 101, 0808.0864.

[21] Y. Imamura and K. Kimura, "On the moduli space of elliptic Maxwell-Chern-Simons theories," 0806.3727.

[22] D. Martelli and J. Sparks, "Moduli spaces of Chern-Simons quiver gauge theories and $\mathrm{AdS}_{4} / \mathrm{CFT}_{3}, "$ Phys. Rev. D78 (2008) 126005, 0808.0912.

[23] A. Hanany and A. Zaffaroni, "Tilings, Chern-Simons Theories and M2 Branes," JHEP 10 (2008) 111, 0808.1244.

[24] A. Hanany, D. Vegh, and A. Zaffaroni, "Brane Tilings and M2 Branes," 0809.1440.

[25] S. Kim, S. Lee, S. Lee, and J. Park, "Abelian Gauge Theory on M2-brane and Toric Duality," Nucl. Phys. B797 (2008) 340-370, 0705.3540.

[26] H. Ooguri and C.-S. Park, "Superconformal Chern-Simons Theories and the Squashed Seven Sphere," 0808.0500.

[27] K. Hosomichi, K.-M. Lee, S. Lee, S. Lee, and J. Park, “N $\mathcal{N} 4$ Superconformal ChernSimons Theories with Hyper and Twisted Hyper Multiplets," JHEP 07 (2008) 091, 0805.3662 .

[28] M. Graña, R. Minasian, M. Petrini, and A. Tomasiello, "A scan for new $\mathcal{N}=1$ vacua on twisted tori," JHEP 05 (2007) 031, hep-th/0609124.

[29] D. Lüst, F. Marchesano, L. Martucci, and D. Tsimpis, "Generalized nonsupersymmetric flux vacua," JHEP 11 (2008) 021, 0807.4540.

[30] K. Behrndt and M. Cvetic, "General $\mathcal{N}=1$ supersymmetric fluxes in massive type IIA string theory," Nucl. Phys. B708 (2005) 45-71, hep-th/0407263. 
[31] L. J. Romans, "Massive N=2a Supergravity in Ten Dimensions," Phys. Lett. B169 (1986) 374.

[32] P. Koerber, D. Lüst, and D. Tsimpis, "Type IIA $\mathrm{AdS}_{4}$ compactifications on cosets, interpolations and domain walls," JHEP 07 (2008) 017, 0804.0614.

[33] M. Graña, R. Minasian, M. Petrini, and A. Tomasiello, "Generalized structures of $\mathcal{N}=1$ vacua," JHEP 11 (2005) 020, hep-th/0505212.

[34] D. Lüst and D. Tsimpis, "Supersymmetric $\mathrm{AdS}_{4}$ compactifications of IIA supergravity," JHEP 02 (2005) 027, hep-th/0412250.

[35] N. J. Hitchin, "The geometry of three-forms in six and seven dimensions," math.dg/0010054.

[36] L. Martucci, "D-branes on general $\mathcal{N}=1$ backgrounds: Superpotentials and Dterms," JHEP 06 (2006) 033, hep-th/0602129. 